Proyecciones

Vol. 27, No 2, pp. 163-177, August 2008.

Universidad Católica del Norte

Antofagasta - Chile

DOI:10.4067/S0716-09172008000200005

\title{
FUNCTIONS OF BOUNDED $(\varphi, p)$ MEAN OSCILLATION
}

\author{
RENÉ ERLÍN CASTILLO \\ OHIO UNIVERSITY, U. S. A. \\ JULIO CÉSAR RAMOS FERNÁNDEZ \\ and \\ EDUARD TROUSSELOT \\ UNIVERSIDAD DE ORIENTE, VENEZUELA \\ Received : November 2006. Accepted : July 2008 \\ Abstract \\ In this paper we extend a result of Garnett and Jones to the case \\ of spaces of homogeneous type.
}

2000 Mathematics Subject Classification : Primary 32A3\%. Secondary 43A85. 


\section{Introduction}

The space of functions of bounded mean oscillation, or $B M O$, naturally arises as the class of functions whose deviation from their means over cubes is bounded. $L_{\infty}$ functions have this property, but there exist unbounded functions with bounded mean oscillation, for instance the function $\log |x|$ is in $B M O$ but it is not bounded. The space $B M O$ shares similar properties with the space $L_{\infty}$ and it often serve as a substitute for it. The space of the functions with bounded mean oscillation $B M O$, is well known for its several applications in real analysis, harmonic analysis and partial differential equations.

The definition of $B M O$ is that $f \in B M O$ if $\sup _{Q} \frac{1}{|Q|} \int_{Q}\left|f(x)-f_{Q}\right| d x=$ $\|f\|_{B M O}<\infty$ where $\mathrm{f}_{Q}=\frac{1}{|Q|} \int_{Q} f(y) d y,|Q|$ is the Lebesgue measure of $Q$ and $Q$ is a cube in $R^{n}$, with sides parallel to the coordinate axes.

In [1] Garnet and Jones gave comparable upper and lower bounds for the distance

$$
\operatorname{dist}\left(f, L_{\infty}\right)=\inf _{g \in L_{\infty}}\|f-g\|_{B M O}
$$

The bounds were expressed in terms of one constant in Jhon-Nirenberg inequality. Jhon and Nirenberg proved in [2] that $f \in B M O$ if and only if there is $\epsilon>0$ and $\lambda_{0}=\lambda_{0}(\epsilon)$ such that

$$
\sup _{Q} \frac{1}{|Q|}\left|\left\{x \in Q:\left|f(x)-f_{Q}\right|>\lambda\right\}\right| \leq e^{-\lambda / \epsilon}
$$

whenever $\lambda>\lambda_{0}=\lambda_{0}(f, \epsilon)$. Indeed, when $f \in B M O$, (1.2) holds with $\epsilon=C\|f\|_{B M O}$, where the constant $c$ depends only on the dimension.

Specifically, setting

$$
\epsilon(f)=\inf \{\epsilon>0: f \text { satisfies }(1.2)\}
$$


Garnett and Jones proved that

$$
A_{1} \epsilon(f) \leq \operatorname{dist}\left(f, L_{\infty}\right) \leq A_{2} \epsilon(f)
$$

where $A_{1}$ and $A_{2}$ are constants depending only on the dimension. Also, they observed that $\operatorname{dist}\left(f, L_{\infty}\right)$ can be related to the growth of

$$
\sup _{Q}\left(\frac{1}{|Q|} \int_{Q}\left|f(x)-f_{Q}\right|^{p} d x\right)^{\frac{1}{p}}
$$

as $p \rightarrow \infty$. This is because

$$
\frac{\epsilon(f)}{e}=\lim _{p \rightarrow \infty} \frac{1}{p}\left(\sup _{Q} \frac{1}{|Q|} \int_{Q}\left|f(x)-f_{Q}\right|^{p} d x\right)^{\frac{1}{p}}
$$

Our latter end is to extend (1.3) to $B M O_{\varphi}^{p}$ (see Preliminaries and Theorem 6.1) on spaces of homogeneous type. Also, we like to point out that (1.3) was announced in [1] without proof. Under the light of Remark 1 (see Preliminaries) we should note that if $|B|=\mu(B)$, then our main result coincide with the result of Garnett and Jones [1].

\section{Spaces of homogeneous type}

Let us begin by recalling the notion of space of homogeneous type.

Definition 2.1. A quasimetric $d$ on a set $X$ is a function $d: X \times X \rightarrow$ $[0, \infty)$ with the following properties:

1. $d(x, y)=0$ if and only if $x=y$.

2. $d(x, y)=d(y, x)$ for all $x, y \in X$.

3. There exists a constant $K$ such that

$$
d(x, y) \leq K[d(x, z)+d(z, y)]
$$

for all $x, y, z \in X$. 
A quasimetric defines a topology in which the balls $B(x, r)=\{y \in X: d(x, y)<r\}$ form a base. These balls may be not open in general; anyway, given a quasimetric $d$, is easy to construct an equivalent quasimetric $d^{\prime}$ such that the $d^{\prime}$-quasimetric balls are open (the existence of $d^{\prime}$ has been proved by using topological arguments in [3]). So we can assume that the quasimetric balls are open. A general method of constructing families $\{B(x, \delta)\}$ is in terms of a quasimetric.

Definition 2.2. A space of homogeneous type $(X, d, \mu)$ is a set $X$ with a quasimetric $d$ and a Borel measure $\mu$ finite on bounded sets such that, for some absolute positive constant $A$ the following doubling property holds

$$
\mu(B(x, 2 r)) \leq A \mu(B(x, r))
$$

for all $x \in X$ and $r>0$.

Next, we are ready to give some example of a space of homogeneous type.

Example 1. Let $X \subset R^{n}, X=\{0\} \cup\{x:|x|=1\}$, put in $X$ the euclidean distance and the following measure $\mu$ : $\mu$ is the usual surface measure on $\{x:|x|=1\}$ and $\mu(\{0\})=1$. Then $\mu$ is doubling so that $(X, d, \mu)$ is a homogeneous space.

Example 2. In $R^{n}$, let $C_{k}(k=1,2, \cdots)$ be the point $\left(k^{k}+1 / 2,0, \cdots, 0\right)$, for $k \geq 2$, let $B_{k}$ be the ball $B\left(C_{k}, 1 / 2\right)$ and $B_{1}=B(0,1 / 2)$. Let $X=\cup_{k=1}^{\infty} B_{k}$ with the euclidean distance and the measure $\mu$ such that $\mu\left(B_{k}\right)=2^{k}$ and on each ball $B_{k}, \mu$ is uniformly distributed.

Claim 1. $\mu$ satisfies the doubling condition. Let $B_{r}=B(P, r)$ with 
$P=\left(P_{1}, \ldots, P_{n}\right)$ and $r>0$.

Case 1. Assume for some $k, B_{k} \subset B_{r}$ and let $k_{0}=\max \left\{k: B_{k} \subset B_{r}\right\}$.

Then certainly $P_{1}+r \leq b_{k_{0}+1}=\left(k_{0}+1\right)^{k_{0}+1}+1$ and $\mu\left(B_{r}\right) \geq 2^{k_{0}}$. But, then

$$
\begin{aligned}
P_{1}+2 r & \leq 2\left(\left(k_{0}+1\right)^{k_{0}+1}+1\right) \\
& \leq\left(k_{0}+2\right)^{k_{0}+2}=a_{k_{0}+2}
\end{aligned}
$$

Therefore $B_{2 r} \subset B_{a_{k_{0}+2}}(0) \equiv B_{0}$. But

$$
\mu\left(B_{0}\right)=\sum_{k=0}^{k_{0}+1} 2^{k} \leq 2^{k_{0}+2} \leq 4 \mu\left(B_{r}\right) .
$$

Hence the doubling condition holds with $A=4$.

Case 2. If for all $k, B_{k} \nsubseteq B_{r}$, then $r<1$ so that $B_{r}$ and $B_{2 r}$ intersect only one ball $B_{k}$. Then the doubling condition holds.

\section{Preliminaries}

In this section, we recall the definition of the space of functions of Bounded $(\varphi, p)$ Mean Oscillation, $B M O_{\varphi}^{(p)}(X)$, where $X$ is a space of homogeneous type (see [4]). Let $\varphi$ be a nonnegative function on $[0, \infty)$. A locally $\mu$ integrable function $f: X \rightarrow R$ is said to belong to the class $\operatorname{BMO}_{\varphi}^{(p)}(X)$, $1 \leq p<\infty$, if

$$
\sup \left(\frac{1}{\mu(B)[\varphi(\mu(B))]^{p}} \int_{B}\left|f(x)-f_{B}\right|^{p} d \mu(x)\right)^{\frac{1}{p}}<\infty,
$$

where the sup is taken over all balls $B \subset X$, and

$$
f_{B}=\frac{1}{\mu(B)} \int_{B} f(y) d \mu
$$


Remark 1. It is not hard to check that the expression

$(3.1)\|f\|_{B M O_{\varphi}^{p}}=\sup _{B}\left(\frac{1}{\mu(B)[\varphi(\mu(B))]^{p}} \int_{B}\left|f(x)-f_{B}\right|^{p} d \mu(x)\right)^{\frac{1}{p}}<\infty$,

define a norm on $\operatorname{BMO}_{\varphi}^{(p)}(X)$. For $\varphi \equiv 1$ and $p=1,\|\cdot\|_{B M O_{\varphi}^{p}}$ coincide with $\|\cdot\|_{B M O}$.

\section{John-Nirenberg inequality on homogeneous type space}

The proof of this theorem follows along the same lines as the proof of [4].

Theorem 4.1. There exist two positive constants $\beta$ and $b$ such that for any $f \in B M O_{\varphi}(X)$ and any ball $B \subset X$, one has

$$
\mu\left(\left\{x \in S:\left|f-f_{B}\right|>\lambda\right\}\right) \leq \beta \exp \left\{-b \lambda /\|f\|_{B M O_{\varphi}}\right\} \mu(B) .
$$

Proof. We follows the standard stopping time argument; that is, we assume that $\lambda$ is large enough and fix some $\lambda_{1}$. Then we study the sets $\left\{x \in S:\left|f(x)-f_{S}\right| \leq \lambda_{1}\right\},\left\{x \in S:\left|f(x)-f_{S}\right| \leq 2 \lambda_{1}\right\}$ up to

$$
\left\{x \in S:\left|f(x)-f_{S}\right| \leq m \lambda_{1} \sim \lambda\right\}
$$

in showing (4.1), we assume $\|f\|_{\varphi}=1$ and fix $S=B(a, R)$. We define a maximal operator associated to $S$ (if we replace $S$ by another ball, then the maximal operator changes)

$$
M_{S} f(x)=\sup _{B \text { ball }, x \in B, B \subset B(a, \alpha R)}\left\{\frac{1}{\varphi(\mu(B)) \mu(B)} \int_{B}\left|f(y)-f_{S}\right| d \mu(y)\right\}
$$

Using a Vitali-type covering lemma, one can prove that

$$
\mu\left(\left\{x: M_{S} f(x)>t\right\}\right) \leq \frac{A}{t} \mu(S)
$$


where $A$ is a constant that depends only on $K$ and $k_{2}$ but not on $S$. Take $\lambda_{0}>A$ and consider the open set $U=\left\{x: M_{S} f(x)>\lambda_{0}\right\}$. We have

$$
\mu(U \cap S) \leq \frac{A}{\lambda_{0}} \mu(S)<\mu(S)
$$

and therefore $S \cap U^{c} \neq \emptyset$. Define

$$
r(x)=\frac{1}{5 K} \operatorname{dist}\left(x, U^{c}\right) .
$$

If $x, y \in S$, then $d(x, y) \leq 2 K R$. Since $S \cap U^{c} \neq \emptyset$, if $x \in S$, we have $r(x) \leq 2 K R /(5 K)=2 R / 5$.

Clearly,

$$
U \cap S \subset \bigcup_{x \in U \cap S} B(x, r(x)) \subset U .
$$

Again by a Vitali-type covering lemma (e. g, see [1, Theorem 3.1]), we can select a finite or countable sequence of disjoint balls $\left\{B\left(x_{j}, r_{j}\right)\right\}$ such that $r_{j}=r_{j}(x)$ and

$$
U \cap S \subset \bigcup_{j} B\left(x_{j}, 4 K r_{j}\right) \subset U .
$$

On the other hand, $B\left(x_{j}, 6 K r_{j}\right) \cap U^{c} \neq \emptyset$ and $B\left(x_{j}, 6 K r_{j}\right) \subset B(a, \alpha R)$ because $6 k r_{j} \leq 12 K R / 5$. Thus, we get

$$
\frac{1}{\varphi\left(\mu\left(B\left(x_{j}, 6 K r_{j}\right)\right)\right) \mu\left(B\left(x_{j}, 6 K r_{j}\right)\right)} \int_{B\left(x_{j}, 6 K r_{j}\right)}\left|f-f_{S}\right| d \mu \leq \lambda_{0}
$$

and consequently, if we write $S_{j}=B\left(x_{j}, 4 K r_{j}\right)$, we obtain

$$
\begin{aligned}
\left|f_{S}-f_{S_{j}}\right| & \leq \frac{1}{\mu\left(S_{j}\right)} \int_{S_{j}}\left|f-f_{S}\right| d \mu \\
& \leq \frac{\varphi\left(S_{j}\right) k_{2}^{2}}{\mu\left(B\left(x_{j}, K r_{j}\right)\right)} \lambda_{0}:=\lambda_{1}
\end{aligned}
$$

because $\mu$ is a doubling measure. 
By differentiation theorem, $\left|f(x)-f_{S}\right| \leq \lambda_{0}$ for $\mu$-a.e. $x \in S \backslash \cup_{j} S_{j}$. Moreover,

$$
\begin{aligned}
\sum \mu\left(S_{j}\right) & \leq k_{2} \sum_{j} \mu\left(B\left(x_{j}, 2 K r_{j}\right)\right) \\
& \leq C \sum_{j} \mu\left(B\left(x_{j}, r_{j}\right)\right) \\
& \leq C \mu(U) \\
& \leq \frac{C A}{\lambda_{0}} \mu(S)
\end{aligned}
$$

Now, we do the same construction for each $S_{j}$. Again $\left|f(x)-f_{S}\right| \leq \lambda_{0}$ for $\mu$-a.e. $x \in S_{j} \backslash \cup_{i} S_{i}^{(2)}$ and therefore for these points

$$
\begin{aligned}
\left|f(x)-f_{S}\right| & \leq\left|f(x)-f_{S_{j}}\right|+\left|f_{S_{j}}-f_{S}\right| \\
& \leq \lambda_{0}+\frac{\varphi\left(S_{j}\right) k_{2}^{2}}{\mu\left(B\left(x_{j}, K r_{j}\right)\right)} \lambda_{0} \\
& \leq \frac{2 \varphi\left(S_{j}\right) k_{2}^{2}}{\mu\left(B\left(x_{j}, K r_{j}\right)\right)} \lambda_{0}
\end{aligned}
$$

taking $\lambda_{0}=2 C A$, it is clear that

$$
\begin{aligned}
\mu\left(\bigcup_{k} S_{k}^{(2)}\right) & \leq \sum_{j} \frac{C A}{\lambda_{0}} \mu\left(S_{j}\right) \\
& \leq\left(\frac{C A}{\lambda_{0}}\right)^{2} \mu(S)=2^{-2} \mu(S) .
\end{aligned}
$$

Continuing in this maner we get $N=1,2, \cdots$ a family of ball $\left\{S_{j}^{N}\right\}$ such that

$$
\mu\left(\bigcup S_{j}^{N}\right) \leq 2^{-N} \mu(S)
$$

finally

$$
\begin{aligned}
\mu\left(\left\{x \in S:\left|f(x)-f_{S}\right|>\lambda\right\}\right) & \leq \mu\left(\left\{x \in S:\left|f(x)-f_{S}\right|>N \lambda_{1}\right\}\right) \\
& \leq \mu\left(\bigcup S_{j}^{N}\right) \leq 2^{-N} \mu(S)=e^{-b \lambda} \mu(S)
\end{aligned}
$$

This complete the proof. 


\section{Completeness}

In this section we state some simple lemmas. The first one is showed by elementary calculations.

Lemma 5.1. Let $B_{0}$ and $B_{1}$ be two balls such that $B_{0} \subset B_{1}$ and $f \in$ $B M O_{\varphi}$. Then there exists a constant $C$ depending on $B_{0}$ and $B_{1}$ such that

$$
\left|f_{B_{0}}-f_{B_{1}}\right| \leq C\|f\|_{B M O_{\varphi}}
$$

Proof. Indeed,

$$
\begin{aligned}
\left|f_{B_{0}}-f_{B_{1}}\right| & =\left|\frac{1}{\mu\left(B_{0}\right)} \int_{B_{0}}\left(f(y)-f_{B_{1}}\right) d \mu(y)\right| \\
& \leq \frac{1}{\mu\left(B_{0}\right)} \int_{B_{1}}\left|f(y)-f_{B_{1}}\right| d \mu(y) \\
& =\frac{\mu\left(B_{1}\right)}{\mu\left(B_{0}\right)} \frac{\varphi(\mu(B))}{\varphi(\mu(B)) \mu\left(B_{1}\right)} \int_{B_{1}}\left|f(y)-f_{B_{1}}\right| d \mu(y) \\
& \leq \frac{\mu\left(B_{1}\right) \varphi(\mu(B))}{\mu\left(B_{0}\right)}\|f\|_{B M O_{\varphi}} .
\end{aligned}
$$

This complete the proof of Lemma 5.1.

Lemma 5.2 (John-Nirenberg type). Let $f \in B M O_{\varphi}^{(p)}(X), 1 \leq p<$ $\infty$, then there exists a constant $C_{p}$ such that

$$
\|f\|_{B M O_{\varphi}} \leq\|f\|_{B M O_{\varphi}^{(p)}} \leq C_{p}\|f\|_{B M O_{\varphi}}
$$

Proof. By Hölder's inequality we have

$$
\frac{1}{\varphi(\mu(B)) \mu(B)} \int_{B}\left|f(y)-f_{B}\right| d \mu(y) \leq \sup _{B}\left(\frac{1}{[\varphi(\mu(B))]^{p} \mu(B)} \int_{B}\left|f(y)-f_{B}\right|^{p} d \mu(y)\right)^{\frac{1}{p}}
$$

for any ball, thus

$$
\|f\|_{B M O_{\varphi}} \leq\|f\|_{B M O_{\varphi}^{(p)}}
$$


On the other hand

$$
\int_{B}\left|f(y)-f_{B}\right|^{p} d \mu(y) \leq \int_{0}^{\infty} p \lambda^{p-1} \mu\left(\left\{x \in B:\left|f(x)-f_{B}\right|>\lambda\right\}\right) d \lambda .
$$

By Theorem 4.1, we obtain

$$
\int_{B}\left|f(y)-f_{B}\right|^{p} d \mu(y) \leq \int_{0}^{\infty} p \lambda^{p-1} \exp \left(-b \lambda /\|f\|_{B M O_{\varphi}}\right) \mu(B) d \lambda .
$$

Therefore

$$
\frac{1}{[\varphi(\mu(B))]^{p} \mu(B)} \int_{B}\left|f(y)-f_{B}\right|^{p} d \mu(y) \leq p \Gamma(p) C\|f\|_{B M O_{\varphi}}
$$

and thus

$$
\|f\|_{B M O_{\varphi}^{(p)}} \leq C_{p}\|f\|_{B M O_{\varphi}}
$$

The Lemma is proved.

Theorem 5.1. $B M O_{\varphi}^{(p)}$ equipped with the norm (3.1) is a Banach space.

Proof. We just need to prove that $B M O_{\varphi}^{(p)}$ is complete. To this end, let us take $B_{1}$ to be the unit ball centered at the origin. Let $f_{k} \in B M O_{\varphi}^{(p)}$, for each $k=1,2,3, \cdots$, such that

$$
\sum_{k=1}^{\infty}\left\|f_{k}\right\|_{B M O_{\varphi}^{(p)}}<\infty
$$

and assume that

$$
\int_{B_{1}} f_{k}(y) d \mu(y)=0 .
$$

Let $B$ be any ball in $X$ and let $B_{2}$ be a ball that contains both $B_{1}$ and $B$, then

$$
\sum_{k=1}^{\infty}\left(\frac{1}{\mu(B)} \int_{B}\left|f_{k}(y)\right|^{p} d \mu(y)\right)^{\frac{1}{p}}=\left(\frac{\mu\left(B_{2}\right)}{\mu(B)}\right)^{\frac{1}{p}} \sum_{k=1}^{\infty}\left(\frac{1}{\mu\left(B_{2}\right)} \int_{B_{2}}\left|f_{k}(y)\right|^{p} d \mu(y)\right)^{\frac{1}{p}} .
$$

By Minkoswki's inequality and by (5.1), we have 


$$
\begin{aligned}
& \sum_{k=1}^{\infty}\left(\frac{1}{\mu(B)} \int_{B}\left|f_{k}(y)\right|^{p} d \mu(y)\right)^{\frac{1}{p}}\left(\frac{\left[\varphi\left(\mu\left(B_{2}\right)\right)\right]^{p} \mu\left(B_{2}\right)}{\mu(B)}\right)^{\frac{1}{p}} \\
\leq & \sum_{k=1}^{\infty}\left(\frac{1}{\left[\varphi\left(\mu\left(B_{2}\right)\right)\right]^{p} \mu\left(B_{2}\right)} \int_{B_{2}}\left|f_{k}(y)-f_{B_{2}}\right|^{p} d \mu(y)\right)^{\frac{1}{p}}+ \\
& +\left(\frac{\mu\left(B_{2}\right)}{\mu(B)}\right)^{\frac{1}{p}} \sum_{k=1}^{\infty}\left(\frac{1}{\mu\left(B_{2}\right)} \int_{B_{2}}\left|\left(f_{k}\right)_{B_{2}}-\left(f_{k}\right)_{B_{1}}\right|^{p} d \mu(y)\right)^{\frac{1}{p}} \\
\leq & \left(\frac{\left[\varphi\left(\mu\left(B_{2}\right)\right]^{p} \mu\left(B_{2}\right)\right.}{\mu(B)}\right)^{\frac{1}{p}} \sum_{k=1}^{\infty}\left[\left\|f_{k}\right\|_{B M O_{\varphi}^{(p)}}+\left(\frac{\mu\left(B_{2}\right)}{\mu(B)}\right)^{\frac{1}{p}}\left|\left(f_{k}\right)_{B_{2}}-\left(f_{k}\right)_{B_{1}}\right|\right] .
\end{aligned}
$$

By Lemma 5.1, we have

$$
\begin{aligned}
& \sum_{k=1}^{\infty}\left(\frac{1}{\mu(B)} \int_{B}\left|f_{k}(y)\right|^{p} d \mu(y)\right)^{\frac{1}{p}} \\
& \leq\left(\frac{\mu\left(B_{2}\right)}{\mu(B)}\right)^{\frac{1}{p}} \sum_{k=1}^{\infty}\left[\left\|f_{k}\right\|_{B M O_{\varphi}}+\left[\varphi\left(\mu\left(B_{2}\right)\right)\right]^{p}\left\|f_{k}\right\|_{B M O_{\varphi}^{(p)}}\right] .
\end{aligned}
$$

By Lemma 5.2 is easy to see that

$$
\begin{aligned}
& \sum_{k=1}^{\infty}\left(\frac{1}{\mu(B)} \int_{B}\left|f_{k}(y)\right|^{p} d \mu(y)\right)^{\frac{1}{p}} \\
& \leq\left(\frac{\mu\left(B_{2}\right)}{\mu(B)}\right)^{\frac{1}{p}} \sum_{k=1}^{\infty}\left(1+\left[\varphi\left(\mu\left(B_{2}\right)\right)\right]^{p}\right)\left\|f_{k}\right\|_{B M O_{\varphi}^{(p)}} .
\end{aligned}
$$

Therefore $\sum_{k=1}^{\infty}\left(\frac{1}{\mu(B)} \int_{B}\left|f_{k}(y)\right|^{p} d \mu(y)\right)^{\frac{1}{p}} \leq \infty$. This means

$$
\left(\frac{1}{\mu(B)}\right)^{\frac{1}{p}} \sum_{k=1}^{\infty}\left\|f_{k}\right\|_{L_{p}}<\infty
$$

and from (5.2), we obtain

$$
f=\lim _{m \rightarrow \infty} \sum_{k=1}^{m} f_{k}, \quad \text { a. e. }
$$

For $f \in L_{p}(B)$, clearly $f_{B}=\sum_{k=1}^{\infty}\left(f_{k}\right)_{B}$.

Finally, we want to show that: 
(a) $f \in B M O_{\varphi}^{(p)}(X)$

(b) $\left\|\sum_{k=1}^{m} f_{k}-f\right\|_{B M O_{\varphi}^{(p)}} \rightarrow 0$ as $m \rightarrow 0$.

To this end, observe that

$$
\begin{aligned}
\left(\frac{1}{\left[\varphi\left(\mu\left(B_{2}\right)\right)\right]^{p} \mu(B)} \int_{B}\left|f(y)-f_{B}\right|^{p} d \mu(y)\right)^{\frac{1}{p}} \\
\quad=\left(\frac{1}{\left[\varphi\left(\mu\left(B_{2}\right)\right)\right]^{p} \mu(B)} \int_{B}\left|\sum_{k=1}^{\infty}\left(f_{k}(y)-\left(f_{k}\right)_{B}\right)\right|^{p} d \mu(y)\right)^{\frac{1}{p}} \\
\quad \leq \sum_{k=1}^{\infty}\left(\frac{1}{\left[\varphi\left(\mu\left(B_{2}\right)\right)\right]^{p} \mu(B)} \int_{B}\left|f_{k}(y)-\left(f_{k}\right)_{B}\right|^{p} d \mu(y)\right)^{\frac{1}{p}} \\
\leq \sum_{k=1}^{\infty}\left\|f_{k}\right\|_{B M O_{\varphi}^{(p)}}<\infty,
\end{aligned}
$$

thus $\|f\|_{B M O_{\varphi}^{(p)}}<\infty$, then $f \in B M O_{\varphi}^{(p)}(X)$. This proves part (a).

On the other hand,

$$
\begin{aligned}
& \left(\frac{1}{\left[\varphi\left(\mu\left(B_{2}\right)\right)\right]^{p} \mu(B)} \int_{B}\left|\left(\sum_{k=1}^{\infty} f_{k}-f\right)(y)-\left(\sum_{k=1}^{m} f_{k}-f\right)_{B}\right|^{p} d \mu(y)\right)^{\frac{1}{p}} \\
& \quad=\left(\frac{1}{\left[\varphi\left(\mu\left(B_{2}\right)\right)\right]^{p} \mu(B)} \int_{B}\left|\sum_{k=m+1}^{\infty}\left(f_{k}(y)-\left(f_{k}\right)_{B}\right)\right|^{p} d \mu(y)\right)^{\frac{1}{p}} \\
& \leq \sum_{k=m+1}^{\infty}\left(\frac{1}{\left[\varphi\left(\mu\left(B_{2}\right)\right)\right]^{p} \mu(B)} \int_{B}\left|f_{k}(y)-\left(f_{k}\right)_{B}\right|^{p} d \mu(y)\right)^{\frac{1}{p}} \\
& \leq \sum_{k=m+1}^{\infty}\left\|f_{k}\right\|_{B M O_{\varphi}^{(p)}} \rightarrow 0, \quad \text { as } m \rightarrow \infty .
\end{aligned}
$$

Hence $\left\|\sum_{k=1}^{m} f_{k}-f\right\|_{B M O_{\varphi}^{(p)}} \rightarrow 0$ as $m \rightarrow 0$. This proves part (b). This completes the proof of the Theorem 5.1.

\section{Main Result}


Theorem 6.1. Let $f \in B M O_{\varphi}^{(p)}$, then there is a constant $\epsilon>0$, such that

$$
\sup \mu\left(\left\{x \in B:\left|f(x)-f_{B}\right|>\lambda\right\}\right) / \mu(B) \leq e^{-\lambda / \epsilon},
$$

where $\lambda>\lambda(\epsilon, f)$. Indeed by Theorem 4.1, we have $\epsilon=C\|f\|_{B M O_{\varphi}^{(p)}}$ and $\lambda(\epsilon, f)=C\|f\|_{B M O_{\varphi}^{(p)}}$. Now let

$$
\epsilon(f)=\inf \{\epsilon:(6.1) \text { holds }\}
$$

Then

$$
\frac{\epsilon(f)}{e \varphi(\mu(B))}=\lim _{p \rightarrow \infty} \frac{1}{p}\|f\|_{B M O_{\varphi}^{(p)}}
$$

Proof. Since

$$
\begin{aligned}
\int_{B(x, r)}\left|f(x)-f_{B}\right|^{p} d \mu(x) & =p \int_{0}^{\infty} \lambda^{p-1} \mu\left(x \in B:\left|f(x)-f_{B}\right|>\lambda\right) d \lambda \\
& \leq p \mu(B) \int_{0}^{\infty} \lambda^{p-1} e^{-\lambda / \epsilon} d \lambda \\
& =\mu(B) \epsilon^{p} \int_{0}^{\infty} u^{p-1} e^{u} d u
\end{aligned}
$$

Thus

$$
\frac{1}{\mu(B)} \int_{B(x, r)}\left|f(x)-f_{B}\right|^{p} d \mu(x) \leq \epsilon^{p} p \Gamma(p) .
$$

Next, we obtain

$$
\frac{1}{p} \sup \left(\frac{1}{[\varphi(\mu(B))]^{p} \mu(B)} \int_{B}\left|f(y)-f_{B}\right|^{p} d \mu(y)\right)^{\frac{1}{p}} \leq \frac{\epsilon[p \Gamma(p)]^{\frac{1}{p}}}{\varphi(\mu(B)) p}
$$

and then,

$$
\text { (6.2 } \lim _{p \rightarrow \infty} \frac{1}{p} \sup \left(\frac{1}{[\varphi(\mu(B))]^{p} \mu(B)} \int_{B}\left|f(y)-f_{B}\right|^{p} d \mu(y)\right)^{\frac{1}{p}} \leq \frac{\epsilon(f)}{e \varphi(\mu(B))} .
$$

On the other hand, if $\epsilon<\epsilon(f)$ then there exists $B_{0} \subset X$, such that

$$
e^{-\lambda / \epsilon} \leq \mu\left(\left\{x \in B_{0}:\left|f(x)-f_{B}\right|>\lambda\right\}\right) / \mu\left(B_{0}\right) .
$$


Thus

$$
p \mu\left(B_{0}\right) \int_{0}^{\infty} \lambda^{p-1} e^{\lambda / \epsilon} d \lambda<p \int_{0}^{\infty} \lambda^{p-1} \mu\left(x \in B:\left|f(x)-f_{B}\right|>\lambda\right) d \lambda
$$

and

$$
\frac{\epsilon[\Gamma(p)]^{\frac{1}{p}}}{\varphi(\mu(B)) p}<\frac{1}{p}\left(\frac{1}{[\varphi(\mu(B))]^{p} \mu(B)} \int_{B}\left|f(y)-f_{B}\right|^{p} d \mu(y)\right)^{\frac{1}{p}} .
$$

It is follows that

$$
{ }_{e \varphi(\mu)} \frac{\epsilon(f)}{e \varphi(B))}<\lim _{p \rightarrow \infty} \frac{1}{p} \sup \left(\frac{1}{[\varphi(\mu(B))]^{p} \mu(B)} \int_{B}\left|f(y)-f_{B}\right|^{p} d \mu(y)\right)^{\frac{1}{p}} \text {. }
$$

Combining (6.2) and (6.3), we obtain the desired result.

Remark 2. Theorem 6.1 together with Lemma 5.2 allow us to estimate the distance from $B M O_{\varphi}^{(p)}$ to $L_{\infty}$ in the other words we can estimate

$$
\inf _{g \in L_{\infty}}\|f-g\|_{B M O_{\varphi}^{(p)}}
$$

with $f \in B M O_{\varphi}^{(p)}$.

Acknowledgments. The authors would like to thank the referee for the useful comments and suggestions which improved the presentation of this paper.

\section{References}

[1] J. Garnett and P. Jones. The distance in $B M O$ to $L_{\infty}$, Ann. of Math., 108, pp. 373-393, (1978).

[2] F. Jhon and L. Nirenberg. On functions of bounded mean oscillation, Comm. on Pure and Appl. Math., XIV, pp. 415-426, (1961). 
[3] R. Marcias and C. Segovia. Lipschitz functions on space of homogeneous type, Avd. Math., 33, pp. 257-270, (1979).

[4] J. Mateu, P. Mattila, A. Nicolau and J. Orobitg. BMO for non-doubling measure, Duke Math. Journal, 102, pp. 533-565, (2000).

\section{René Erlín Castillo}

Department of Mathematics

Ohio University

Athens,

Ohio 45701

e-mail address: rcastill@math.ohiou.edu

\section{Julio César Ramos Fernández}

Departamento de Matemáticas

Universidad de Oriente

6101 Cumaná,

Edo. Sucre, Venezuela

e-mail : jramos@sucre.udo.edu.ve

and

\section{Eduard Trousselot}

Departamento de Matemáticas

Universidad de Oriente

6101 Cumaná,

Edo. Sucre, Venezuela

e-mail : eddycharles2007@hotmail.com 